(C) by R. Oldenbourg Verlag, München

\title{
Crystal structure of 2,4,6-tri-tert-butylphenylazide, $\left(\mathrm{C}_{4} \mathrm{H}_{9}\right)_{3} \mathrm{C}_{6} \mathrm{H}_{2} \mathrm{~N}_{3}$
}

\author{
K. Peters, E.-M. Peters
}

Max-Planck-Institut für Festkörperforschung. Heisenbergstraße 1. D-70506 Stuttgan. Germany

\section{J. Balthasar and H. Quast}

Institut für Organische Chemie der Universität Würzburg, Am Hubland, D-97074 Würzburg, Germany

Received March 10, 1998. CSD-No. 409274
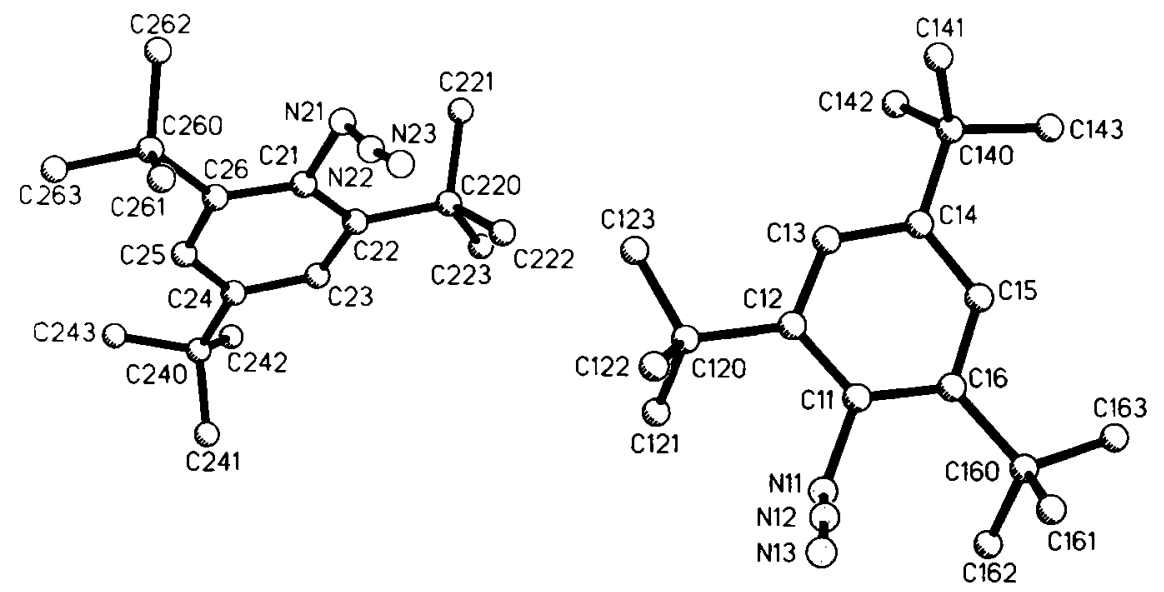

Source of material: The title compound was prepared, according to ref. 1-3, by conversion of 2,4,6-tri-tert-butylaniline with an excess of gaseous nitric oxide into the diazonium nitrate (see ref. 4) followed by treatment with hydrazine hydrate. Introduction of nitric oxide in a stream of argon into a solution of the aniline in ether at $273 \mathrm{~K}-278$ $\mathrm{K}$ yielded a pale yellow precipitate which was not isolated. Addition of hydrazine hydrate to the suspension after $0.5 \mathrm{~h}$, followed by stirring at $293 \mathrm{~K}-298 \mathrm{~K}$ for $4 \mathrm{~h}$ and evaporation of the solvent yielded a green, solid product (melting point range $388 \mathrm{~K}-396 \mathrm{~K}$ ) consisting of the title compound, 1,3,5-tri-tert-butylbenzene, and mainly 2,4,6tri-tert-butylnitrosobenzene. Flash chromatography on silica gel with petroleum ether separated the nitroso compound (39\% yield, mp 440 $\mathrm{K}$, see ref. 5). Cyclic medium pressure liquid chromatography of the remaining two-component mixture on silica gel with petroleum ether ( 6 repetitive cycles) separated 1,3,5-tri-tert-butylbenzene ( $8 \%$ yield, $\mathrm{mp} 400 \mathrm{~K}-401 \mathrm{~K}$ ) and the title compound (8\% yield) which gave colorless crystals, mp $395 \mathrm{~K}-396 \mathrm{~K}$, on recrystallization from petroleum ether.

Separation of the title compound from 1,3,5-tri-tert-butylbenzene, which inevitably arises in similar amounts, is very difficult, and the melting point ( $401 \mathrm{~K}-402 \mathrm{~K}$ ), reported in ref. 3 for the title compound, is actually that of 1,3,5-tri-tert-butylbenzene.

The unit cell of the title compound contains two pairs of different molecules which are distinguished by slightly different torsional angles. Due to the tert-butyl groups at the ortho positions, the azido group adopts a perpendicular conformation relative to the ring plane.

$\mathrm{C}_{18 \mathrm{H}_{29} \mathrm{~N} 3}$, triclinic, $P \overline{1}$ (No. 2), $a=11.745(4) \AA, b=17.113(5) \AA$, $c=9.983(3) \AA, \alpha=97.86(2)^{\circ}, \beta=107.96(2)^{\circ}, \gamma=98.67(2)^{\circ}$,

$V=1850.7 \AA^{3}, Z=4, R(F)=0.085, R_{\mathrm{w}}(F)=0.079$.
Table 1. Parameters used for the X-ray data collection

\begin{tabular}{ll}
\hline Crystal: & colorless plate, size $0.4 \times 0.8 \times 0.2 \mathrm{~mm}$ \\
Wavelength: & Mo $K_{\alpha}$ radiation $(0.71073 \AA)$ \\
$\mu:$ & $0.60 \mathrm{~cm}^{-1}$ \\
Diffractometer: & Siemens R3m/V \\
Scan mode: & Wyckoff \\
$T_{\text {measurement: }}$ & $293 \mathrm{~K}$ \\
$2 \theta_{\max }:$ & $55^{\circ}$ \\
$\mathrm{N}(\text { } k \mathrm{kl})_{\text {unique: }}$ & 8499 \\
Criterion for $F_{\mathrm{o}}:$ & $F_{\mathrm{o}}>3 \sigma\left(F_{\mathrm{o}}\right)$ \\
$\mathrm{N}(\text { param })_{\text {refined: }}$ & 434 \\
Program: & SHELXTL-plus \\
\hline
\end{tabular}

Table 2. Final atomic coordinates and displacement parameters (in $\AA^{2}$ )

\begin{tabular}{|c|c|c|c|c|c|}
\hline Atom & Site & $x$ & $y$ & $z$ & $U_{\text {iso }}$ \\
\hline$H(13)$ & $2 i$ & $0.3026(3)$ & $0.3431(2)$ & $-0.0503(3)$ & 0.08 \\
\hline$H(15)$ & $2 i$ & $0.2428(2)$ & $0.5664(2)$ & $-0.0803(3)$ & 0.08 \\
\hline $\mathrm{H}(12 \mathrm{~A})$ & $2 i$ & $0.3629(3)$ & $0.4010(2)$ & $0.4084(4)$ & 0.08 \\
\hline $\mathrm{H}(12 \mathrm{~B})$ & $2 i$ & $0.4567(3)$ & $0.3795(2)$ & $0.3338(4)$ & 0.08 \\
\hline $\mathrm{H}(12 \mathrm{C})$ & $2 i$ & $0.3942(3)$ & $0.3150(2)$ & $0.4004(4)$ & 0.08 \\
\hline H(12D) & $2 i$ & $0.1369(3)$ & $0.3210(2)$ & $0.2854(4)$ & 0.08 \\
\hline$H(12 E)$ & $2 i$ & $0.1794(3)$ & $0.2394(2)$ & $0.2589(4)$ & 0.08 \\
\hline $\mathrm{H}(12 \mathrm{~F})$ & $2 i$ & $0.1012(3)$ & $0.2719(2)$ & $0.1287(4)$ & 0.08 \\
\hline$H(12 G)$ & $2 i$ & $0.2674(5)$ & $0.2372(2)$ & $0.0315(4)$ & 0.08 \\
\hline $\mathrm{H}(12 \mathrm{H})$ & $2 i$ & $0.3388(5)$ & $0.2200(2)$ & $0.1817(4)$ & 0.08 \\
\hline $\mathrm{H}(12 \mathrm{I})$ & $2 i$ & $0.4024(5)$ & $0.2835(2)$ & $0.1143(4)$ & 0.08 \\
\hline $\mathrm{H}(14 \mathrm{~A})$ & $2 i$ & $0.1907(4)$ & $0.3272(3)$ & $-0.3037(4)$ & 0.08 \\
\hline $\mathrm{H}(14 \mathrm{~B})$ & $2 i$ & $0.2098(4)$ & $0.3673(3)$ & $-0.4294(4)$ & 0.08 \\
\hline$H(14 C)$ & $2 i$ & $0.1194(4)$ & $0.3943(3)$ & $-0.3538(4)$ & 0.08 \\
\hline$H(14 D)$ & $2 i$ & $0.4227(3)$ & $0.3702(3)$ & $-0.1832(4)$ & 0.08 \\
\hline H(14E) & $2 i$ & $0.4807(3)$ & $0.4625(3)$ & $-0.1453(4)$ & 0.08 \\
\hline
\end{tabular}


Table 2. (Continued)

\begin{tabular}{llllll}
\hline Atom & Site & \multicolumn{1}{l}{$\boldsymbol{x}$} & \multicolumn{1}{l}{$z$} & $U_{\text {iso }}$ \\
\hline H(14F) & $2 i$ & $0.4323(3)$ & $0.4166(3)$ & $-0.3055(4)$ & 0.08 \\
H(14G) & $2 i$ & $0.2178(4)$ & $0.5366(2)$ & $-0.3154(4)$ & 0.08 \\
H(14H) & $2 i$ & $0.3122(4)$ & $0.5074(2)$ & $-0.3824(4)$ & 0.08 \\
H(14I) & $2 i$ & $0.3579(4)$ & $0.5593(2)$ & $-0.2266(4)$ & 0.08 \\
H(16A) & $2 i$ & $0.0572(3)$ & $0.5750(2)$ & $0.2294(5)$ & 0.08 \\
H(16B) & $2 i$ & $0.0244(3)$ & $0.5860(2)$ & $0.0694(5)$ & 0.08 \\
H(16C) & $2 i$ & $0.0593(3)$ & $0.6615(2)$ & $0.1934(5)$ & 0.08 \\
H(16D) & $2 i$ & $0.2879(4)$ & $0.6143(2)$ & $0.3822(4)$ & 0.08 \\
H(16E) & $2 i$ & $0.2791(4)$ & $0.7001(2)$ & $0.3467(4)$ & 0.08 \\
H(16F) & $2 i$ & $0.3775(4)$ & $0.6559(2)$ & $0.3126(4)$ & 0.08 \\
H(16G) & $2 i$ & $0.1644(4)$ & $0.6618(2)$ & $-0.0151(4)$ & 0.08 \\
H(16H) & $2 i$ & $0.3055(4)$ & $0.6856(2)$ & $0.0681(4)$ & 0.08 \\
H(16I) & $2 i$ & $0.2161(4)$ & $0.7295(2)$ & $0.1235(4)$ & 0.08 \\
H(23) & $2 i$ & $0.9028(3)$ & $0.1638(2)$ & $0.5424(3)$ & 0.08 \\
H(25) & $2 i$ & $0.7651(3)$ & $-0.0585(2)$ & $0.5824(3)$ & 0.08 \\
H(22A) & $2 i$ & $0.7038(5)$ & $0.0986(3)$ & $0.0840(4)$ & 0.08 \\
H(22B) & $2 i$ & $0.8457(5)$ & $0.1257(3)$ & $0.1589(4)$ & 0.08 \\
H(22C) & $2 i$ & $0.7694(5)$ & $0.1865(3)$ & $0.0899(4)$ & 0.08 \\
H(22D) & $2 i$ & $0.5788(4)$ & $0.1749(2)$ & $0.1970(5)$ & 0.08 \\
H(22E) & $2 i$ & $0.6599(4)$ & $0.2611(2)$ & $0.2264(5)$ & 0.08 \\
H(22F) & $2 i$ & $0.6299(4)$ & $0.2273(2)$ & $0.3525(5)$ & 0.08 \\
& & & & &
\end{tabular}

Table 2. (Continued)

\begin{tabular}{llllll}
\hline Atom & Site & $x$ & $y$ & $z$ & $U_{\text {iso }}$ \\
\hline H(22G) & $2 i$ & $0.8584(4)$ & $0.2694(3)$ & $0.4473(5)$ & 0.08 \\
H(22H) & $2 i$ & $0.8745(4)$ & $0.2799(3)$ & $0.3002(5)$ & 0.08 \\
H(22I) & $2 i$ & $0.9442(4)$ & $0.2227(3)$ & $0.3898(5)$ & 0.08 \\
H(24A) & $2 i$ & $0.9109(5)$ & $0.1601(4)$ & $0.8115(4)$ & 0.08 \\
H(24B) & $2 i$ & $0.9760(5)$ & $0.1185(4)$ & $0.9361(4)$ & 0.08 \\
H(24C) & $2 i$ & $0.8368(5)$ & $0.0866(4)$ & $0.8478(4)$ & 0.08 \\
H(24D) & $2 i$ & $1.0734(4)$ & $0.1588(6)$ & $0.7045(6)$ & 0.08 \\
H(24E) & $2 i$ & $1.0619(4)$ & $0.0658(6)$ & $0.6592(6)$ & 0.08 \\
H(24F) & $2 i$ & $1.1320(4)$ & $0.1092(6)$ & $0.8190(6)$ & 0.08 \\
H(24G) & $2 i$ & $0.8949(7)$ & $-0.0354(3)$ & $0.7753(6)$ & 0.08 \\
H(24H) & $2 i$ & $1.0350(7)$ & $-0.0095(3)$ & $0.8632(6)$ & 0.08 \\
H(24I) & $2 i$ & $0.9909(7)$ & $-0.0353(3)$ & $0.6956(6)$ & 0.08 \\
H(26A) & $2 i$ & $0.4262(3)$ & $-0.0707(2)$ & $0.2629(5)$ & 0.08 \\
H(26B) & $2 i$ & $0.4521(3)$ & $-0.0771(2)$ & $0.4244(5)$ & 0.08 \\
H(26C) & $2 i$ & $0.3925(3)$ & $-0.1543(2)$ & $0.3035(5)$ & 0.08 \\
H(26D) & $2 i$ & $0.5504(4)$ & $-0.1177(2)$ & $0.1234(4)$ & 0.08 \\
H(26E) & $2 i$ & $0.5237(4)$ & $-0.2018(2)$ & $0.1653(4)$ & 0.08 \\
H(26F) & $2 i$ & $0.6590(4)$ & $-0.1574(2)$ & $0.1981(4)$ & 0.08 \\
H(26G) & $2 i$ & $0.5985(4)$ & $-0.1542(2)$ & $0.5208(5)$ & 0.08 \\
H(26H) & $2 i$ & $0.6991(4)$ & $-0.1718(2)$ & $0.4547(5)$ & 0.08 \\
H(26I) & $2 i$ & $0.5672(4)$ & $-0.2256(2)$ & $0.3905(5)$ & 0.08 \\
& & & & &
\end{tabular}

Table 3. Final atomic coordinates and displacement parameters (in $\AA^{2}$ )

\begin{tabular}{|c|c|c|c|c|c|c|c|c|c|c|}
\hline Atom & Site & $x$ & $y$ & $z$ & $U_{11}$ & $U_{22}$ & $U_{33}$ & $U_{12}$ & $U_{13}$ & $U_{23}$ \\
\hline$N(11)$ & $2 i$ & $0.2105(2)$ & $0.4745(2)$ & $0.3090(2)$ & $0.063(2)$ & $0.067(2)$ & $0.045(1)$ & $0.012(1)$ & $0.024(1)$ & $0.012(1)$ \\
\hline $\mathbf{N}(12)$ & $2 i$ & $0.1055(3)$ & $0.4561(2)$ & $0.3072(3)$ & $0.078(2)$ & $0.081(2)$ & $0.063(2)$ & $0.027(2)$ & $0.037(2)$ & $0.029(1)$ \\
\hline $\mathbf{N}(13)$ & $2 i$ & $0.0129(3)$ & $0.4405(2)$ & $0.3210(4)$ & $0.092(3)$ & $0.150(3)$ & $0.130(3)$ & $0.045(2)$ & $0.071(2)$ & $0.071(3)$ \\
\hline$C(11)$ & $2 i$ & $0.2319(2)$ & $0.4671(2)$ & $0.1721(3)$ & $0.045(2)$ & $0.051(2)$ & $0.033(1)$ & $0.009(1)$ & $0.014(1)$ & $0.006(1)$ \\
\hline$C(12)$ & $2 i$ & $0.2637(2)$ & $0.3967(2)$ & $0.1201(3)$ & $0.052(2)$ & $0.049(2)$ & $0.042(2)$ & $0.014(1)$ & $0.016(1)$ & $0.011(1)$ \\
\hline$C(13)$ & $2 i$ & $0.2826(3)$ & $0.3915(2)$ & $-0.0112(3)$ & $0.061(2)$ & $0.054(2)$ & $0.046(2)$ & $0.021(1)$ & $0.018(1)$ & $0.004(1)$ \\
\hline$C(14)$ & $2 i$ & $0.2738(2)$ & $0.4534(2)$ & $-0.0885(3)$ & $0.044(2)$ & $0.062(2)$ & $0.038(1)$ & $0.012(1)$ & $0.014(1)$ & $0.007(1)$ \\
\hline$C(15)$ & $2 i$ & $0.2476(2)$ & $0.5229(2)$ & $-0.0287(3)$ & $0.044(2)$ & $0.052(2)$ & $0.044(2)$ & $0.008(1)$ & $0.015(1)$ & $0.013(1)$ \\
\hline$C(16)$ & $2 i$ & $0.2275(2)$ & $0.5335(2)$ & $0.1032(3)$ & $0.041(2)$ & $0.045(2)$ & $0.041(1)$ & $0.007(1)$ & $0.011(1)$ & $0.004(1)$ \\
\hline$C(120)$ & $2 i$ & $0.2828(3)$ & $0.3266(2)$ & $0.2038(3)$ & $0.079(2)$ & $0.056(2)$ & $0.060(2)$ & $0.028(2)$ & $0.025(2)$ & $0.021(2)$ \\
\hline$C(121)$ & $2 i$ & $0.3828(3)$ & $0.3590(2)$ & $0.3509(4)$ & $0.090(3)$ & $0.096(3)$ & $0.074(2)$ & $0.034(2)$ & $0.021(2)$ & $0.045(2)$ \\
\hline$C(122)$ & $2 i$ & $0.1637(3)$ & $0.2870(2)$ & $0.2215(4)$ & $0.103(3)$ & $0.062(2)$ & $0.098(3)$ & $0.014(2)$ & $0.037(2)$ & $0.033(2)$ \\
\hline$C(123)$ & $2 i$ & $0.3264(5)$ & $0.2608(2)$ & $0.1245(4)$ & $0.156(4)$ & $0.081(3)$ & $0.098(3)$ & $0.069(3)$ & $0.056(3)$ & $0.041(2)$ \\
\hline$C(140)$ & $2 i$ & $0.2958(3)$ & $0.4423(2)$ & $-0.2329(3)$ & $0.058(2)$ & $0.081(2)$ & $0.040(2)$ & $0.018(2)$ & $0.022(1)$ & $0.012(2)$ \\
\hline$C(141)$ & $2 i$ & $0.1952(4)$ & $0.3764(3)$ & $-0.3397(4)$ & $0.093(3)$ & $0.134(4)$ & $0.047(2)$ & $0.001(3)$ & $0.027(2)$ & $-0.003(2)$ \\
\hline$C(142)$ & $2 i$ & $0.4188(3)$ & $0.4205(3)$ & $-0.2149(4)$ & $0.081(3)$ & $0.146(4)$ & $0.059(2)$ & $0.042(3)$ & $0.037(2)$ & $0.017(2)$ \\
\hline$C(143)$ & $2 i$ & $0.2936(4)$ & $0.5183(2)$ & $-0.2954(4)$ & $0.124(3)$ & $0.120(3)$ & $0.060(2)$ & $0.041(3)$ & $0.054(2)$ & $0.030(2)$ \\
\hline$C(160)$ & $2 i$ & $0.2050(3)$ & $0.6153(2)$ & $0.1652(3)$ & $0.057(2)$ & $0.043(2)$ & $0.053(2)$ & $0.009(1)$ & $0.018(1)$ & $0.003(1)$ \\
\hline$C(161)$ & $2 i$ & $0.0751(3)$ & $0.6092(2)$ & $0.1667(5)$ & $0.075(3)$ & $0.056(2)$ & $0.130(3)$ & $0.024(2)$ & $0.041(2)$ & $0.010(2)$ \\
\hline$C(162)$ & $2 i$ & $0.2958(4)$ & $0.6487(2)$ & $0.3159(4)$ & $0.098(3)$ & $0.059(2)$ & $0.077(2)$ & $0.009(2)$ & $0.018(2)$ & $-0.009(2)$ \\
\hline$C(163)$ & $2 i$ & $0.2261(4)$ & $0.6791(2)$ & $0.0781(4)$ & $0.137(4)$ & $0.050(2)$ & $0.097(3)$ & $0.021(2)$ & $0.057(3)$ & $0.017(2)$ \\
\hline $\mathbf{N}(21)$ & $2 i$ & $0.5721(3)$ & $0.0231(2)$ & $0.1813(3)$ & $0.069(2)$ & $0.082(2)$ & $0.059(2)$ & $0.018(2)$ & $0.003(1)$ & $0.015(1)$ \\
\hline $\mathbf{N}(22)$ & $2 i$ & $0.4730(3)$ & $0.0432(2)$ & $0.1770(3)$ & $0.080(2)$ & $0.073(2)$ & $0.083(2)$ & $0.016(2)$ & $0.009(2)$ & $0.019(2)$ \\
\hline $\mathbf{N}(23)$ & $2 i$ & $0.3768(3)$ & $0.0578(2)$ & $0.1547(5)$ & $0.079(3)$ & $0.119(3)$ & $0.184(4)$ & $0.042(2)$ & $0.016(3)$ & $0.042(3)$ \\
\hline$C(21)$ & $2 i$ & $0.6655(3)$ & $0.0345(2)$ & $0.3214(3)$ & $0.051(2)$ & $0.052(2)$ & $0.043(2)$ & $0.010(1)$ & $0.010(1)$ & $0.009(1)$ \\
\hline$C(22)$ & $2 i$ & $0.7534(3)$ & $0.1065(2)$ & $0.3714(3)$ & $0.055(2)$ & $0.048(2)$ & $0.052(2)$ & $0.011(1)$ & $0.019(1)$ & $0.013(1)$ \\
\hline$C(23)$ & $2 i$ & $0.8425(3)$ & $0.1145(2)$ & $0.5045(3)$ & $0.052(2)$ & $0.051(2)$ & $0.057(2)$ & $0.004(1)$ & $0.017(1)$ & $0.004(1)$ \\
\hline$C(24)$ & $2 i$ & $0.8482(3)$ & $0.0541(2)$ & $0.5856(3)$ & $0.049(2)$ & $0.067(2)$ & $0.044(2)$ & $0.018(2)$ & $0.016(1)$ & $0.007(1)$ \\
\hline$C(25)$ & $2 i$ & $0.7615(3)$ & $-0.0161(2)$ & $0.5283(3)$ & $0.058(2)$ & $0.056(2)$ & $0.055(2)$ & $0.016(2)$ & $0.021(2)$ & $0.020(1)$ \\
\hline$C(26)$ & $2 i$ & $0.6684(2)$ & $-0.0291(2)$ & $0.3955(3)$ & $0.049(2)$ & $0.049(2)$ & $0.051(2)$ & $0.009(1)$ & $0.017(1)$ & $0.011(1)$ \\
\hline$C(220)$ & $2 i$ & $0.7581(3)$ & $0.1750(2)$ & $0.2854(4)$ & $0.078(2)$ & $0.056(2)$ & $0.075(2)$ & $0.015(2)$ & $0.030(2)$ & $0.028(2)$ \\
\hline$C(221)$ & $2 i$ & $0.7691(5)$ & $0.1428(3)$ & $0.1408(4)$ & $0.169(4)$ & $0.106(3)$ & $0.102(3)$ & $0.058(3)$ & $0.081(3)$ & $0.064(3)$ \\
\hline $\mathrm{C}(222)$ & $2 i$ & $0.6469(4)$ & $0.2135(2)$ & $0.2652(5)$ & $0.118(3)$ & $0.063(2)$ & $0.118(3)$ & $0.034(2)$ & $0.041(3)$ & $0.036(2)$ \\
\hline $\mathrm{C}(223)$ & $2 i$ & $0.8698(4)$ & $0.2426(3)$ & $0.3628(5)$ & $0.112(4)$ & $0.091(3)$ & $0.150(4)$ & $-0.008(3)$ & $0.033(3)$ & $0.069(3)$ \\
\hline$C(240)$ & $2 i$ & $0.9476(3)$ & $0.0662(2)$ & $0.7313(3)$ & $0.054(2)$ & $0.088(2)$ & $0.046(2)$ & $0.022(2)$ & $0.008(1)$ & $0.009(2)$ \\
\hline$C(241)$ & $2 i$ & $0.9149(5)$ & $0.1097(4)$ & $0.8429(4)$ & $0.122(4)$ & $0.261(7)$ & $0.049(2)$ & $0.102(4)$ & $-0.003(2)$ & $-0.019(3)$ \\
\hline$C(242)$ & $2 i$ & $1.0642(4)$ & $0.1079(6)$ & $0.7346(6)$ & $0.083(4)$ & $0.62(2)$ & $0.109(5)$ & $-0.086(7)$ & $-0.037(3)$ & $0.147(7)$ \\
\hline$C(243)$ & $2 i$ & $0.9727(7)$ & $-0.0084(3)$ & $0.7751(6)$ & $0.31(1)$ & $0.206(8)$ & $0.136(5)$ & $0.152(8)$ & $-0.138(6)$ & $-0.037(5)$ \\
\hline$C(260)$ & $2 i$ & $0.5781(3)$ & $-0.1113(2)$ & $0.3379(3)$ & $0.060(2)$ & $0.048(2)$ & $0.073(2)$ & $0.005(1)$ & $0.020(2)$ & $0.011(2)$ \\
\hline$C(261)$ & $2 i$ & $0.4502(3)$ & $-0.1039(2)$ & $0.3325(5)$ & $0.082(3)$ & $0.071(2)$ & $0.139(4)$ & $-0.003(2)$ & $0.050(3)$ & $0.007(2)$ \\
\hline $\mathrm{C}(262)$ & $2 i$ & $0.5783(4)$ & $-0.1502(2)$ & $0.1930(4)$ & $0.102(3)$ & $0.069(3)$ & $0.108(3)$ & $-0.010(2)$ & $0.037(3)$ & $-0.015(2)$ \\
\hline$C(263)$ & $2 i$ & $0.6136(4)$ & $-0.1720(2)$ & $0.4336(5)$ & $0.131(4)$ & $0.057(3)$ & $0.145(4)$ & $-0.010(3)$ & $0.008(3)$ & $0.033(3)$ \\
\hline
\end{tabular}




\section{References}

1. Quast. H.: Balthasar. J.: Unpublished results.

2. Rigaudy, J.: Vernieres, J. C.: Nitric oxide as diazotization agent. Application to 2,4,6-trisubstituted anilines. Compt. Rend. Acad. Sci.; Paris, Ser. C 261 (1965) 5516-5519.

3. Rigaudy. J.: Vermieres. J. C.: Efficient method of preparing $N$-(2,4,6-tritert-butylphenyl)hydrazine. Extension to the preparation of $N$-(2,4,6-triphenylphenyl)hydrazine. Compt. Rend. Acad. Sci.: Paris, Ser. C 266 (1968) 828-831.
4. Barclay, L. R. C.; Briggs, A. G.; Briggs, W. E.; Dust, J. M.; Gray, J. A.: Electron spin resonance and product studies of the dediazoniation reaction. Canad. J. Chem. 57 (1979) 2172-2179.

5. Okazaki, R.; Hosogai, T.; Iwadare. E.; Hashimoto, M.; Inamoto, N.: Preparation of sterically hindered nitrosobenzenes. Bull. Chem. Soc. Jpn. 42 (1969) $3611-3612$.

6. Sheldrick, G. M.: Program Package SHELXTL-plus. Release 4.1. Siemens Analytical X-Ray Instruments Inc.. Madison (W1 53719). USA 1990. 